\title{
TEM Study of Current-Induced Domain Wall Motion in Cylindrical Nanowires: Towards 3D Magnetic Memory Devices
}

\author{
Sergei Lopatin ${ }^{1}$, Yurii P. Ivanov², Jurgen Kosel $^{3}$ and Andrey Chuvilin ${ }^{4,5}$. \\ 1. Core Labs, King Abdullah University of Science \& Technology, Thuwal, Saudi Arabia \\ 2. Erich Schmid Institute of Materials Science, Austrian Academy of Sciences, Leoben, Austria \\ 3. Computer, Elec, Math Sciences \& Eng, King Abdullah University of Science \& Technology, Thuwal, \\ Saudi Arabia \\ 4. CIC nanoGUNE, Donostia-San Sebastian, Spain \\ 5. IKERBASQUE, Basque Foundation for Science, Bilbao, Spain.
}

Modern information storage technology strives toward realizing 3D devices. A very promising idea for 3D magnetic memory, called vertical race-track memory, proposed in 2008, was to manipulate information by utilizing current-driven domain wall (DW) motion in magnetic nanowires (NWs) [1]. Specifically, magnetic domains, separated by DWs, represent bits of information, whereas DW position and motion (i.e. information storage and transfer) can be controlled by spin-polarized electric currents. The main challenge in realizing this idea comes from the standard micro-fabrication methods, which are highly effective for fabrication planar devices but very limited in capabilities when it comes to out-ofplane structures. One way to increase the memory density could be stacking of storage layers in a single device. This complicates significantly the fabrication process even for a small number of layers. Hence, there is a need for efficient alternative fabrication concepts.

Cylindrical magnetic nanowires are attractive materials for the next generation of 3D data storage devices, due to potentially very high speed of domain wall (DW) motion and their efficient fabrication in dense arrays. Cylindrical NWs can be fabricated efficiently inside nanoporous templates and provide various unique properties. Recently, we have proposed a storage media composed of dense arrays of cylindrical multisegmented $\mathrm{Ni} / \mathrm{Co}$ nanowires arranged vertically to the substrate and fabricated by a simple electrodeposition technique. We demonstrated that the interfaces between $\mathrm{Ni}$ and Co segments create a periodic energy landscape and act as pinning sites for the DW propagation [2]. The magnetic structure of the DWs is of 3D vortex type with a Bloch point at its center. The next step in realizing a race-track memory with cylindrical nanowires is to deliberately and reproducibly move the DWs from one pinning site to another.

For planar magnetic nanowires the DW motion driven by the spin-polarized electric currents is well studied and intrinsic limitations of such systems are well understood. This includes the Walker breakdown phenomenon, which was shown to limit the propagation speed of the DW (a crucial parameter for fast memories) and the problem of unwanted DW pinning at imperfections in planar structures. For non-planar (cylindrical) nanowires the probability of DW pinning at random structural imperfections significantly decreases, and theoretical models show that 3D DWs do not suffer from the Walker breakdown phenomenon. However, current-driven DW motion in cylindrical nanowires was not addressed until recently [3].

In this work current induced dynamics of 3D magnetic vortex domain walls in cylindrical $\mathrm{Ni} / \mathrm{Co}$ nanowires with periodic variation of $\mathrm{Ni}(\mathrm{fcc})$ and $\mathrm{Co}(\mathrm{hcp})$ segments was accessed theoretically by micromagnetic simulations, and experimentally by Lorentz microscopy, and recently developed 
modification of differential phase contrast, based on the idea of virtual bright field unitary detector (VBF-DPC) [4]. Experimental part was performed with a Titan 60-300 TEM (FEI Co) equipped with a high brightness electron gun (x-FEG), Lorentz lens in combination with image Cs corrector and Fischione HAADF STEM detector. The microscope was operated at $200 \mathrm{keV}$ acceleration voltage with objective lens set close to $0 \%$ to allow reduced and adjustable magnetic field environment. We have demonstrated by direct observation (Fig.1) with Cs corrected Lorentz TEM imaging that spin-polarized electric current can control reversible motion of 3D vortex domain walls [3], which travel with a velocity of a few hundred meters per second. This finding constitutes a key step in establishing highdensity, fast 3D memory devices based on vertical arrays of cylindrical magnetic nanowires.

\section{References:}

[1] S. S. P. Parkin et al, Science 320 (2008), p. 190.

[2] Yu. P. Ivanov et al, ACS nano 10 (5) (2016), p. 5326.

[3] Yu. P. Ivanov et al, ACS Appl. Mater. Interfaces 9 (20) (2017), p. 16741.

[4] S. Lopatin et al, Ultramicroscopy 162, (2016), p. 74.
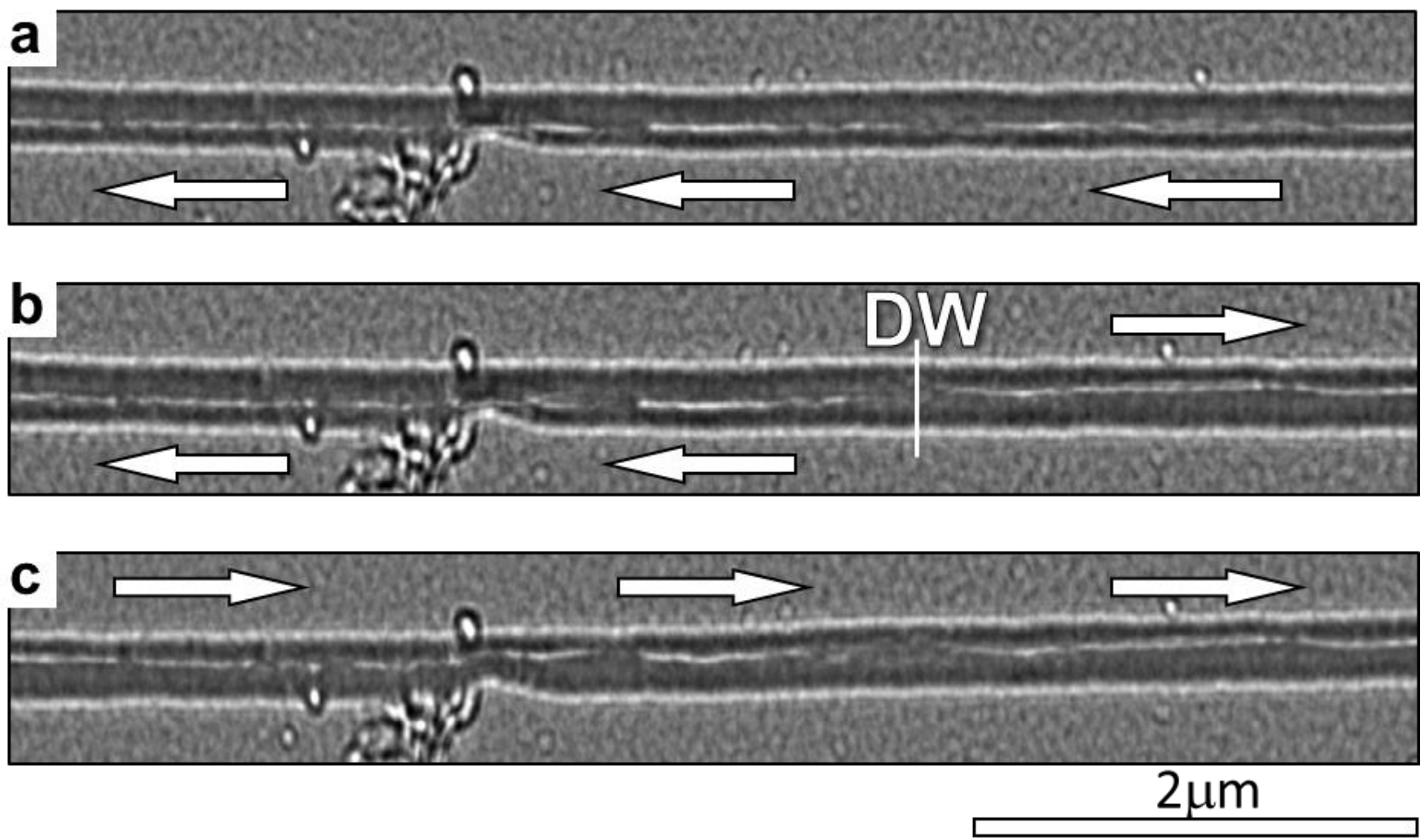

Figure 1. Over focused Lorentz images of the Ni/Co nanowire: a) the initial state with magnetization along the NW pointing to the left, b) the DW pinned at the interface between Ni and Co segments, c) DW moved with electric current and magnetization switch in opposite direction. 